\title{
Pollution and Oxidative Stress in Schoolchildren
}

\author{
GARY E HATCH \\ Research Toxicologist, US Environmental Protection Agency, Mail Drop B143-01, Research Triangle Park, NC 27713, USA.
}

I $\mathrm{n}$ this issue of Indian Pediatrics, investigators from Belgrade and Pancevo report on a comparison of blood plasma chemistry between children living in a petrochemicalpolluted city and a nearby more rural (and supposedly cleaner) city in Serbia(1). Of 24 measurements made on the plasma, three showed significance which appeared to be related to increased oxidative stress in the polluted city: (i) elevated (26\%) malondialdehyde (MDA), (ii) decreased (14\%) activity of superoxide dismutase (SOD), and (iii) decreased activity of paraoxonase-1 (PON1) among a genetic subgroup of the children.

The 'MDA assay' actually measures a host of different products of oxidized lipids and other biomolecules and has been shown to correlate in humans with cardiovascular and other diseases(2). The SOD assay of blood plasma is less wellunderstood as an oxidative stress marker; however, it is clear that lower activity of this enzyme could predispose to oxidative stress(3). Lower plasma activities of the enzyme, PON1, could also predispose to oxidative stress. PON1 inhibits oxidation of low-density lipoproteins and hydrolyzes lipid peroxides in atherosclerotic lesions (although it is probably best known for its role in detoxifying organophosphorus pesticides). PON1 genotypes and enzyme activities have been associated with numerous health outcomes in recent epidemiological studies. A small subset $(<10 \%)$ of the children studied (of a total of 124) possessed the riskassociated RR phenotype of PON1, and among these children living in the polluted city, the PON1 activity was decreased (significantly) to about $60 \%$ of that observed in children from the less polluted city.
These findings are significant because they add to a body of data from epidemiological studies spanning a variety of different disease and exposure conditions - all indicating measurable oxidative stress markers associated with higher pollutant exposures. Some of the measures used are somewhat crude from a chemistry standpoint, but they are notable in that they tend to point in the same direction. It should also be remembered that any chemical measure that is detectable in blood plasma has already been diluted substantially from what would have been detectable at the tissue sites where it originates (such as airways and lung in the case of air pollutants), and it has also survived effective mechanisms for its elimination. As the paper correctly points out, increased concentrations of particulate matter and other air pollutants have been implicated in many epidemiological studies examining cardiovascular morbidity and mortality.

It would be of future value to improve the linkage between biological effects and pollutant levels and exposure. This can be done by ensuring that epidemiological studies are accompanied by state-of-the-art monitoring of the ambient pollution. Also, the blood samples taken represent considerable work and any efforts to improve archiving of such samples and making them available to worldwide investigators should be encouraged.

The inclusion by the authors of markers for susceptible genetic subgroups is laudable because it shows an appreciation for the fact that humans are not all the same in their ability to compensate for injury induced by environmental pollutants. This inability to compensate may be more important in the long-term than the extent of the initial injury. 
Funding: None.

Competing interests: None stated.

\section{REFERENCES}

1. Vujovic A, Kotur-Stevuljevic J, Kornic D, Spasic $\mathrm{S}$, Spasojevic-Kalimanovska V, BogavacStanojevic N, et al. Oxidative stress and antioxidative defense in schoolchildren residing in a petrochemical industry environment. Indian Pediatr 2010; 47: 233-239.
2. Walter MF, Jacob RF, Jeffers B, Ghadanfar MM, Preston GM, Buch J, et al. Serum levels of thiobarbituric acid reactive substances predict cardiovascular events in patients with stable coronary artery disease: a longitudinal analysis of the PREVENT study. J Am Coll Cardiol 2004; 44: 1996-2002.

3. Cabrera EJ, Valezi AC, Delfino VDA, Lavado EL, Barbosa DS. Reduction in plasma levels of inflammatory and oxidative stress indicators after Roux-en-Y gastric by pass. Obes Surg 2010; 20: 42-49. 